\title{
Imitation Proteoglycans Improve Toughness of Double Network
}

\section{Hydrogels}

\author{
Junhua Wei, Siheng Su, Jilong Wang, Jingjing Qiu* \\ * Corresponding Author: Dr. Jingjing Qiu, Department of Mechanical Engineering, Texas Tech \\ University, Box 41021, Lubbock TX 79409, Tel: (806)834-8076, Email: Jenny.Qiu@ @tu.edu
}

\begin{abstract}
:
Although tough double network hydrogel (DN gel) has been fabricated for years, the practical applications haven't achieved. One of the reasons is the limited polymer choices. The recent researches indicated that the addition of the Proteoglycans (PG), a heavily glycosylated protein, can make soft DN gel tough. However, the extract of natural PG and synthesize of its substitute are expensive and time consuming. In this work, chondroitin sulfate coated carbon nanotube (CS-T), a nanostructure biomimic PG, was synthesized to make soft hydrogel tough. $295 \%$ and $360 \%$ enhancements have been found on compressive strength and toughness after adding small amount of CS-T, respectively. As the PG plays a critical role in the cartilage to resist compression and maintain microstructure, this novel nanostructure paves an affordable way to produce substitutes for load-bearing tissue and multi-functional hydrogel.

Key words: biomaterials, mechanical testing, chemical synthesis, nanostructures.
\end{abstract}

\section{Introduction}

Hydrogels are water-swollen, three-dimensional crosslinked macromolecular networks. Due to their good biocompatibility, responsiveness to various stimuli, low surface friction, and environmental friendliness [1-5], the hydrogels, especially made from the biopolymers like agar, hyaluronan, alginate, and collagen, are considered innovative materials for bio-applications such as scaffolds and tissues [6-8]. However, for years, the low toughness nature of the hydrogel 
restricts their potential applications as tissue substitute [9]. In order to improve their mechanical properties, several special hydrogel structures have been synthesized, like slide-ring, nanocomposite, tri-block copolymers, hydrophobic modified, tetra-PEG gels, and macromolecular microsphere composite hydrogels [10-12]. Among them, one of the most notable approaches is the double-network gel (DN gel) structure which presents both rubber-like robustness and high water content. The optimized DN gels show excellent hardness (modulus: 0.1-1.0 MPa), strength (tensile fracture stress: 1-10 MPa; strain 100-3000\%), and toughness (tearing energy: 100-4500 J/m2) which are comparable to or even superb than the natural cartilage [13-17]. However, very limited polymers can be used to fabricate tough DN gel because of its unique structure.

Tough DN gel consists of two polymer networks with contrasting physical properties: a densely crosslinked strong polyelectrolyte network (rigid and brittle skeleton: N1) in low concentrations and a sparsely crosslinked neutral polymer network (soft and ductile substance: N2) in high concentrations $[18,19]$. Because of the highly charged N1 extents the DN gel, the material of the $\mathrm{N} 1$ is required to be polyelectrolyte. Although more and more charged biopolymers were used to fabricate DN gel [20,21], neutral biopolymers are still not applicable for DN gel. In this case, an approach to introduce more biopolymers into DN gel is demanded for variable applications.

During recent years, several approaches have been carried out. Suekama et al. have produced tough DN hydrogels by using chemically modified methacrylate chondroitin sulfate as the N1 [22]. However, its disadvantages, like complicated modifying process and unfavorable changes in the biochemical properties caused by the chemical crosslinking, restrict its practical applications. The so-called molecular stent method is a more preferred method due to its simple 
processing and little influence on biopolymers [23, 24]. In this method, a natural biopolyelectrolyte is dispersed within the $\mathrm{N} 1$ which is made by unmodified, chemical inert and neutral biopolymer. The bio-polyelectrolyte enhances osmotic pressure to extend and toughen the N1 network gel while the N2 network prevents hydrogel breaking. Although previous researches have shown some promising results $[23,24]$, in order to have DN gel with excellent mechanical properties, a special bio-polyelectrolyte, chondroitin sulfate proteoglycans (PG), is required which is expensive to purchase and difficult to extract. The addition of other synthesized polyelectrolytes and bio-polyelectrolytes, like chondroitin sulfate (CS) and sodium hyaluronate, exhibited very limited mechanical improvement and serious leaking due to their low charge density and small structures, respectively [24]. A simple method to produce biomimic PG is required for clinic applications.

In the PG, large numbers of the CS are anchored onto a protein backbone to form a large structure with high charge density. Herein, chondroitin sulfate coated carbon nanotube (CS-T), biomimic by the PG, is fabricated. Large numbers of the CS are anchored onto functionalized carbon nanotube (CNT). The limited modification on the CS (1 to 2 sites within $\sim 150 \mathrm{k}$ Da structure) ensures its bio-properties while the functionalization on the CNT offers large amounts of reaction sites. Due to the large numbers of CS attaching and the charge repelling between CS chains, this structure is spread into micrometer size and is capable to entangle with multiple N1 chains, which can prevent leaking. As the CS is much cheaper than the PG and the synthesis processing of CS-T is simple, this structure is promising to fabricate tough hydrogel with different functions by using the most suitable polymers.

\section{Materials and Methods}




\subsection{Materials}

Chondroitin sulfate (CS, 220k Da), allylamine, nitric acid, allyl glycidyl ether (AGE), acrylamide (AA), N, N'-methylenebis (acrylamide) (MBAA), sodium borate buffer, sodium borate buffer, N-(3-Dimethylaminopropyl)-N'-ethylcarbodiimide (EDC), and ammonium persulfate (APS) were purchased from Sigma Aldrich.

\subsection{Allyl Glycidyl Ether modification Chondroitin sulfate (AGE-CS)}

In order to add reaction site to the CS, the AGE was used. AGE, an epoxide and vinyl containing monomer, can react with amines at an alkaline pH. Briefly, AGE was added into 10 $\mathrm{mg} / \mathrm{ml}$ of $\mathrm{CS}$ in sodium borate buffer (SBB, 0.1M, $\mathrm{pH}$ 9.4) to form 1000:1 molar ratio of AGE to CS $[25,26]$. After stirring this solution for $24 \mathrm{~h}$, the AGE modified CS (AGE-CS) was purified by dialysis (12kDa MWCO) for 7 days.

\subsection{Allyl Functionalized CNT (ACNT)}

The functionalization of the CNT is similar to our previous research [27]. Briefly, CNT was reflux at $120{ }^{\circ} \mathrm{C}$ for $8 \mathrm{hr}$ with $7 \mathrm{M}$ nitric acid to form Oxidized CNT (O-CNT). O-CNT was washed with hydrochloride acid and water for times. The allyl groups were added onto CNT through amide reaction with allylamine catalyzed by EDC.

\subsection{Chondroitin sulfate coated Carbon Nanotube (CS-T)}

The Chondroitin Sulfate was coated onto Carbon Nanotube through a free radical reaction. Briefly, after tip-sonicate $5 \mathrm{ml}$ of $1 \mathrm{mg} / \mathrm{ml} \mathrm{ACNT}$ for $30 \mathrm{~min}, 100 \mathrm{ml}$ of the $10 \mathrm{mg} / \mathrm{ml}$ purified AGE-CS solution was added. $5 \mathrm{mg}$ of the APS was then added into this solution after nitrogen gas bubbling. The mixture was stirred, heated at $65^{\circ} \mathrm{C}$, and protected by the $\mathrm{N}_{2}$

atmosphere for $10 \mathrm{~h}$. The CS-T was purified by dialysis for 3 days and collected by 
centrifugation. The PCS (poly (allyl glycidyl ether modified Chondroitin sulfate)) was synthesized similar with CS-T without adding ACNT. The synthesis is presented in Fig. 1.

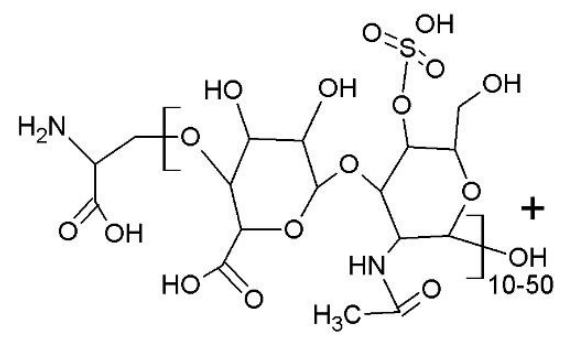

CS<smiles>C=CCOCC1CO1</smiles>

AGE

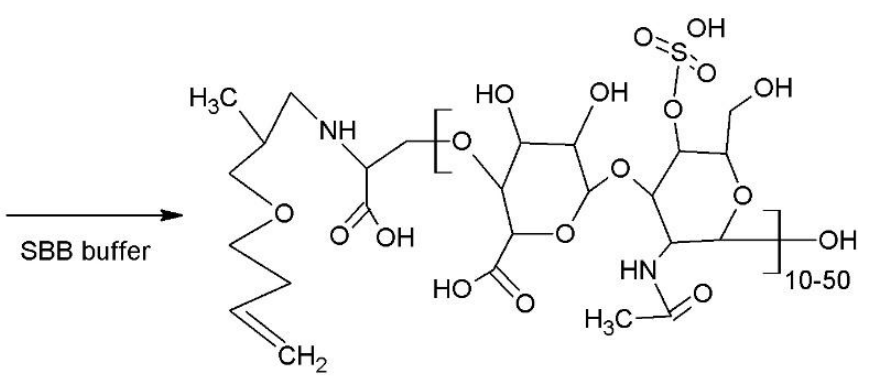

AGE-CS

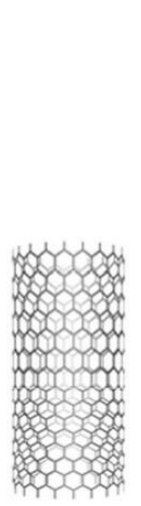

CNT

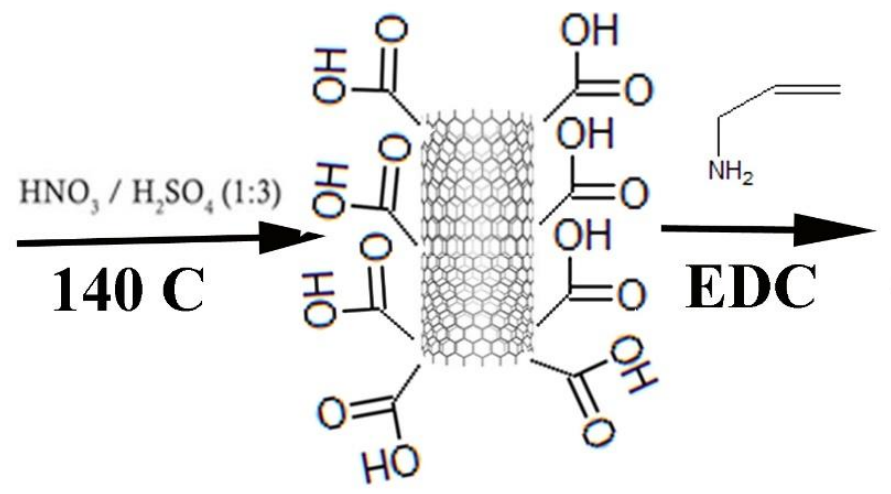

O-CNT

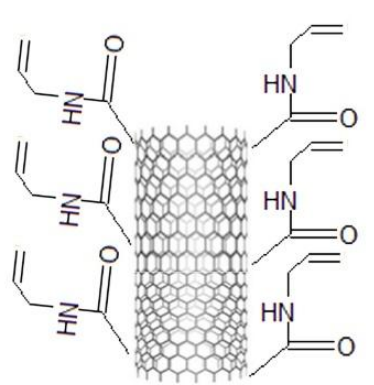

ACNT

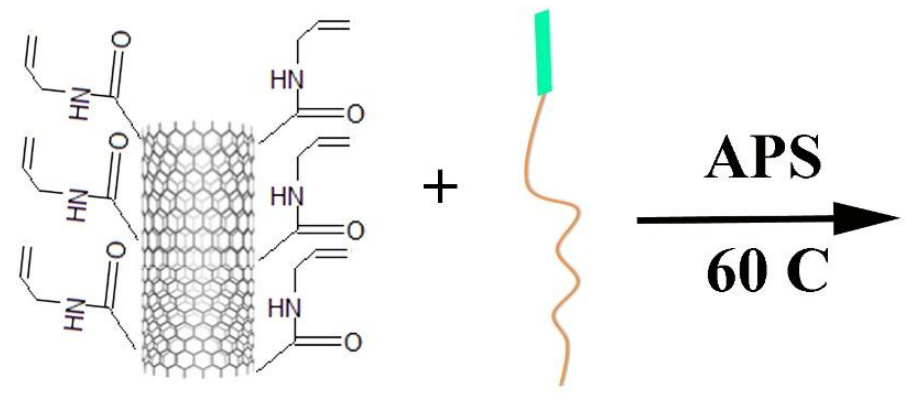

ACNT

AGE-CS

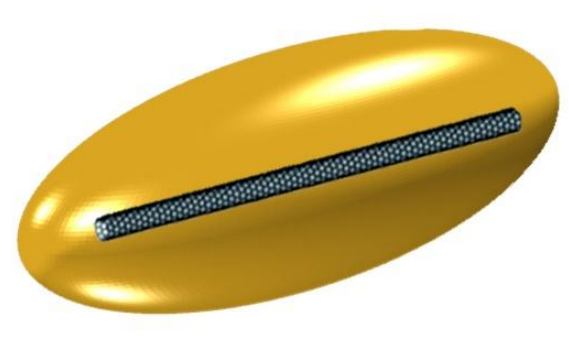

CS-T 
Fig. 1. The scheme of fabrication Chondroitin Sulfate coated Carbon Nanotube (CS-T) through double bond reaction.

\subsection{Double Network hydrogels (DN gel)}

Table 1. Sample, Stent, and Compositions of DN gels

\begin{tabular}{|c|c|c|c|}
\hline Sample & Stent & wt $^{(\mathrm{a})}$ & $\mathrm{x} 1-\mathrm{y} 1 / \mathrm{x} 2-\mathrm{y} 2^{(\mathrm{c})}$ \\
\hline Control & - & - & $1-2 / 4-0.02$ \\
\hline D(CS1) & CS & 1 & $1-2 / 4-0.02$ \\
\hline D(CS3) & CS & 3 & $1-2 / 4-0.02$ \\
\hline D(CS6) & CS & 6 & $1-2 / 4-0.02$ \\
\hline D(PCS1) & PCS & 1 & $1-2 / 4-0.02$ \\
\hline D(PCS3) & PCS & 3 & $1-2 / 4-0.02$ \\
\hline D(PCS6) & PCS & 6 & $1-2 / 4-0.02$ \\
\hline D(CS-T1) & CST & 1 & $1-2 / 4-0.02$ \\
\hline D(CS-T3) & CST & 3 & $1-2 / 4-0.02$ \\
\hline D(CS-T6) & CST & 6 & $1-2 / 4-0.02$ \\
\hline D(CS6+ACNT) & CS+ACNT & $6+0.03^{(b)}$ & $1-2 / 4-0.02$ \\
\hline
\end{tabular}

Note: ${ }^{(a)}$ the $w t \%$ of the stent is the weight ratio of stent in the dried weight of the first network (N1). ${ }^{(b)} 6$ wt $\%$ of CS with $0.03 \mathrm{wt} \%$ of ACNT was added to form D(CS6+ACNT). ${ }^{(c)}$ In $\mathrm{x} 1-\mathrm{y} 1 / \mathrm{x} 2-\mathrm{y} 2, \mathrm{x}$ and $\mathrm{y}$ denote the AA monomer concentration (M) and MBAA crosslinker density (mol\%) with respect to the monomer, respectively; the subscripts 1 and 2 denote the $\mathrm{N} 1$ and $\mathrm{N} 2$ network, respectively.

In this work, DN gels were synthesized via a two-step sequential free-radical polymerization with molecular stent [24]. Different DN gels with different molecular stent (D(CS) with CS, D(PCS) with PCS, and D(CS-T) with CS-T) were prepared. In the first step, 1 M AA, $4 \mathrm{~mol} \%$ of the MBAA, and $0.1 \mathrm{~mol} \%$ thermo initiator (APS) were dissolved in pure water with different wt $\%$ of different stents. The mixture was then polymerized at $60^{\circ} \mathrm{C}$ for $10 \mathrm{~h}$ to form the N1 network gel: N1. In the second step, N1 was soaked for $1 \mathrm{~d}$ in AA swelling solution (contained $4 \mathrm{M} \mathrm{AA}, 0.02 \mathrm{~mol} \% \mathrm{MBAA}$, and $0.01 \mathrm{~mol} \%$ APS). Then the swollen N1 was polymerized again at $60{ }^{\circ} \mathrm{C}$ for $10 \mathrm{~h}$. The polymerized DN gels were submerged in pure water for $2 \mathrm{~d}$ to remove unreacted reagents. In order to investigate the influence of stent structure, $6 \mathrm{wt} \% \mathrm{CS}$ and $0.03 \mathrm{wt} \%$ CNT were added into the DN gel. The compositions of different DN 
gels are shown in Table 1. In order to invest the influence of additional stents, the control sample was fabricated using the same process without adding any stent.

The swelling degree, $\mathrm{q}$, of the hydrogel was calculated by using the expression: $Q=\frac{w_{\text {swell }}}{w_{0}} \times 100 \%$, where $w_{\text {swell }}$ and $w_{0}$ denote the weights of the swollen and the as prepared hydrogel, respectively. 5 sets of $w_{\text {swell }}$ and $w_{0}$ were measured for each sample.

The mechanical properties of the DN gels were investigated by unconfined uniaxial compressive measurements with $10 \%$ strain per min speed. 5 cylinders with $10 \mathrm{mn}$ diameter and $5 \mathrm{~mm}$ thickness were fabricated and tested for each sample using a commercial universal test machine (SHIMADZU, AGS-X). The engineering compressive stress was calculated by $\sigma_{c}=\operatorname{load} / \pi R^{2}$, where $\mathrm{R}$ is the original radius of the cylindrical specimen. The engineering stress is defined as the change in the height relative to the original height: $\varepsilon_{c}=100 \% \times\left(h_{0}-h\right) / h_{0}$. Stress and strain between $\varepsilon_{c}=0$ and 10 were used to calculate the

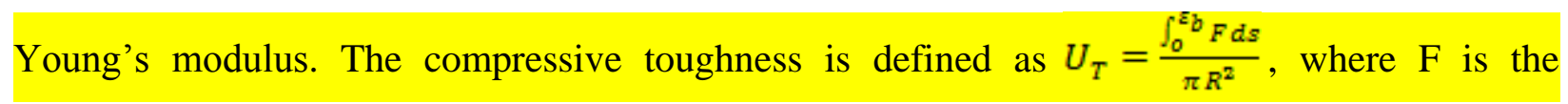
compressive load, $\mathrm{s}$ is the compressive displacement to the corresponding strain, and the $\varepsilon_{b}$ is stress at break[28]. The molecular weights of CS and PCS were tested by the light scattering method with 5 different concentrations for linear fitting (Malvern, DLS ZS90). The morphologies of the ACNT and CS-T were imaged by TEM using $75 \mathrm{kV}$ voltage (Transmission Electronic Microscopy, HITACHI T8100). The chemical structures were studied by FTIR using ATR mold (Fourier Transform Infrared Spectroscopy, Nicolet iS10). The UV-vis absorption spectrum is measured on Infinite M1000 Pro plate reader by solution method (Tecan).

\subsection{Leaking}


$1 \mathrm{~g} \mathrm{~N} 1 \mathrm{gel}$ of each sample (S(CS6), S(PCS6), and S(CTS6), S denoted as single network hydrogel) were polymerized. Instead of merging them into the AA monomer solution, these hydrogels were swollen in $50 \mathrm{ml}$ water for 7 days. The water in each beaker was analyzed by the UV absorption. The relative concentrations of CS in the water were measured by their peak intensity around $260 \mathrm{~nm}$ in their UV absorption spectra.

\subsection{In Vitro Studies}

A brain cancer cell line U87-MG (ATCC, HTB-14) was used to test hydrogel's biocompatibility. $3 \times 104$ cells/well of U87-MG cells were seeded in a 24 -well polystyrene plate and cultured in $350 \mu \mathrm{l}$ of growth medium composed of Eagle's Minimum Essential Medium (EMEM) with 10\% fetal bovine serum (FBS) and $100 \mathrm{IU} / \mathrm{ml}$ penicillin and streptomycin for 24 hours. Then the medium was replaced by another $350 \mu 1$ of culture medium in which was immersed with hydrogels ( $2 \mathrm{~mm}$ in diameter and $2 \mathrm{~mm}$ in thickness) for 7 days. After 24,48 , or $72 \mathrm{~h}$, hydrogel was removed and the number of cells is quantified using a crystal violet staining method[29].

\section{Results}

\subsection{Allyl Glycidyl Ether functionalized Chondroitin Sulfate}

The AGE-CS was first investigated by the FTIR spectra (Fig. 2 (a)). The peaks of CS included: $\mathrm{S}=\mathrm{O}\left(1230 \mathrm{~cm}^{-1}\right)$, C-O-S $\left(812,927,730 \mathrm{~cm}^{-1}\right),-\mathrm{OH}\left(3200-3600 \mathrm{~cm}^{-1}\right)$, amide/carboxyl $\left(1621 \mathrm{~cm}^{-1}\right), \mathrm{C}-\mathrm{C}\left(1130 \mathrm{~cm}^{-1}\right), \mathrm{C}-\mathrm{H}\left(1550 \mathrm{~cm}^{-1}\right), \mathrm{C}-\mathrm{O}\left(1052 \mathrm{~cm}^{-1}\right)[30,31]$. The shoulder peaks at 912 (epoxide), 1094 (ether stretching), 2857 (CH2=CH2 stretching), and 1647 (alkyl stretching)

$\mathrm{cm}^{-1}$ in the FTIR spectrum of AGE-CS indicated the successful attachment of AGE onto CS [25, 32]. 
The molecular weights of AGE-CS and PCS were measured by DLS which also indicated the successful attachment (Fig. 2(b) and (c)). The reciprocal of the intersection at X axis and the linear fitting line indicated the MW of the tested polymer. As calculated, the MW of AGE-CS was $16 \mathrm{kDa}$ while the MW of PCS was $5431 \mathrm{kDa}$. On average, 350 AGE-CS were polymerized into a small bottle brush polymer through the double bond on the AGE. Due to the large negative charge and steric hinder effect, larger PCS can't be achieved by directly polymerizing AGE-CS.

The FTIR spectra and the MW measured by DLS exhibited the AGE has been attached onto CS and the double bond on the AGE is activatable for polymerization.
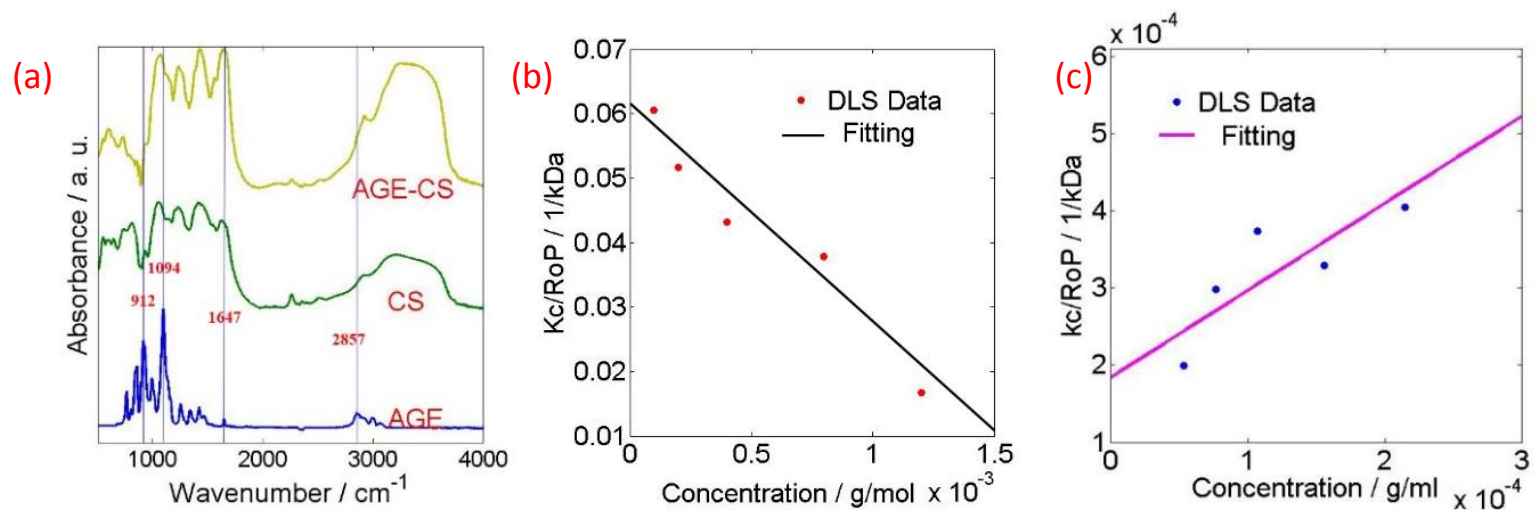

Fig. 2. The FTIR spectra of AGE, CS, and AGE-CS (a), and the molecular weights of AGE-CS

(b) and PCS (c).

\subsection{Allyl Functionalized Carbon Nanotube}

The FTIR spectra of O-CNT and ACNT were compared in Fig. 3. In the O-CNT, the peak at $3444 \mathrm{~cm}^{-1}$ indicated the $-\mathrm{OH}$ moieties and $-\mathrm{OH}$ in carboxyl groups. The peaks around $1740 \mathrm{~cm}^{-1}$ and $1620 \mathrm{~cm}^{-1}$ represented the $\mathrm{C}=\mathrm{O}$ bands and ketone in carboxyl functional groups, respectively. Peaks ranging of $900-1300 \mathrm{~cm}^{-1}$ represented the $\mathrm{C}-\mathrm{O}$ bonds in various chemical surroundings. A band at $1620 \mathrm{~cm}^{-1}$ was due to aromatic and unsaturated structural of $\mathrm{C}=\mathrm{C}$ bonds and the bands at $1380 \mathrm{~cm}^{-1}$ and $1460 \mathrm{~cm}^{-1}$ were due to $\mathrm{OH}$ in-plane deformation and $\mathrm{C}-\mathrm{O}$ moiety in C-O-C, respectively[33, 34]. In the ACNT, the $1380 \mathrm{~cm}^{-1}$, which represent the $\mathrm{C}=\mathrm{C}$ bonds are 
increased. The nitrogen groups in the allylamine was confirmed by the peaks at $800 \mathrm{~cm}^{-1}$ and $1560 \mathrm{~cm}^{-1}$, which represented the primary amine groups and the stretching of amine groups, respectively[27]. The morphology of the ACNT was presented by TEM image in Fig. 3 (b).
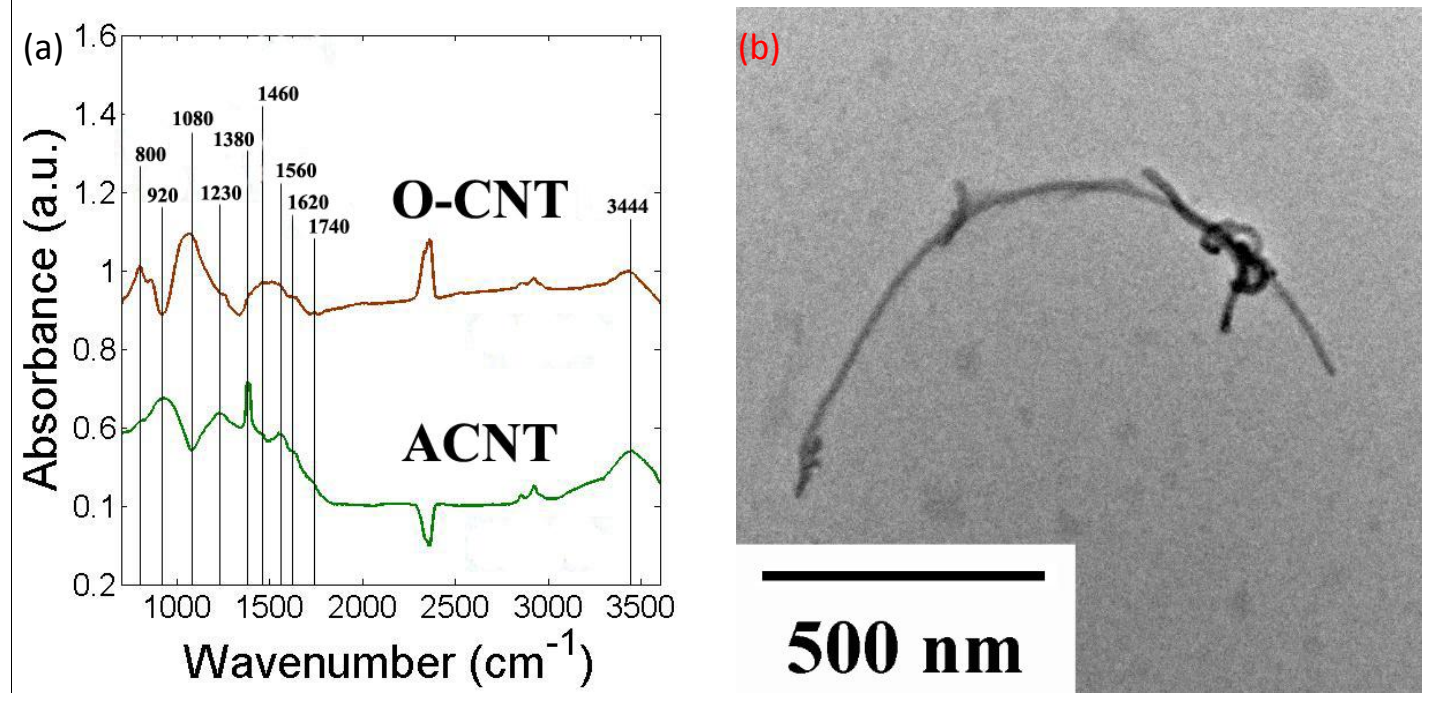

Fig. 3. (a) FTIR spectra of ACNT and O-CNT and the (b) TEM image of the ACNT.

\subsection{Chondroitin Sulfate coated Carbon Nanotube}

The attachment of the CS on CNT was confirmed by TEM images and FTIR spectrum. The CNT as the backbone is wrapped within the CS chains. Because the TEM samples were prepared by dropping and drying. The large extended CS-T structure was spread to form a large 2D thin sheet on the grid due to the large number of small CS chains attached onto the CNT. The FTIR spectrum also exhibited the existence of peaks for CS ( $\mathrm{S}=\mathrm{O}: 1230 \mathrm{~cm}^{-1}$, C-O-S: 980,720 $\mathrm{cm}^{-1}$ ) and CNT (-COOH:1740 $\mathrm{cm}^{-1}$ and $1620 \mathrm{~cm}^{-1}$, C-O-C: $1380 \mathrm{~cm}^{-1}$ and $\left.1460 \mathrm{~cm}^{-1}\right)$. 

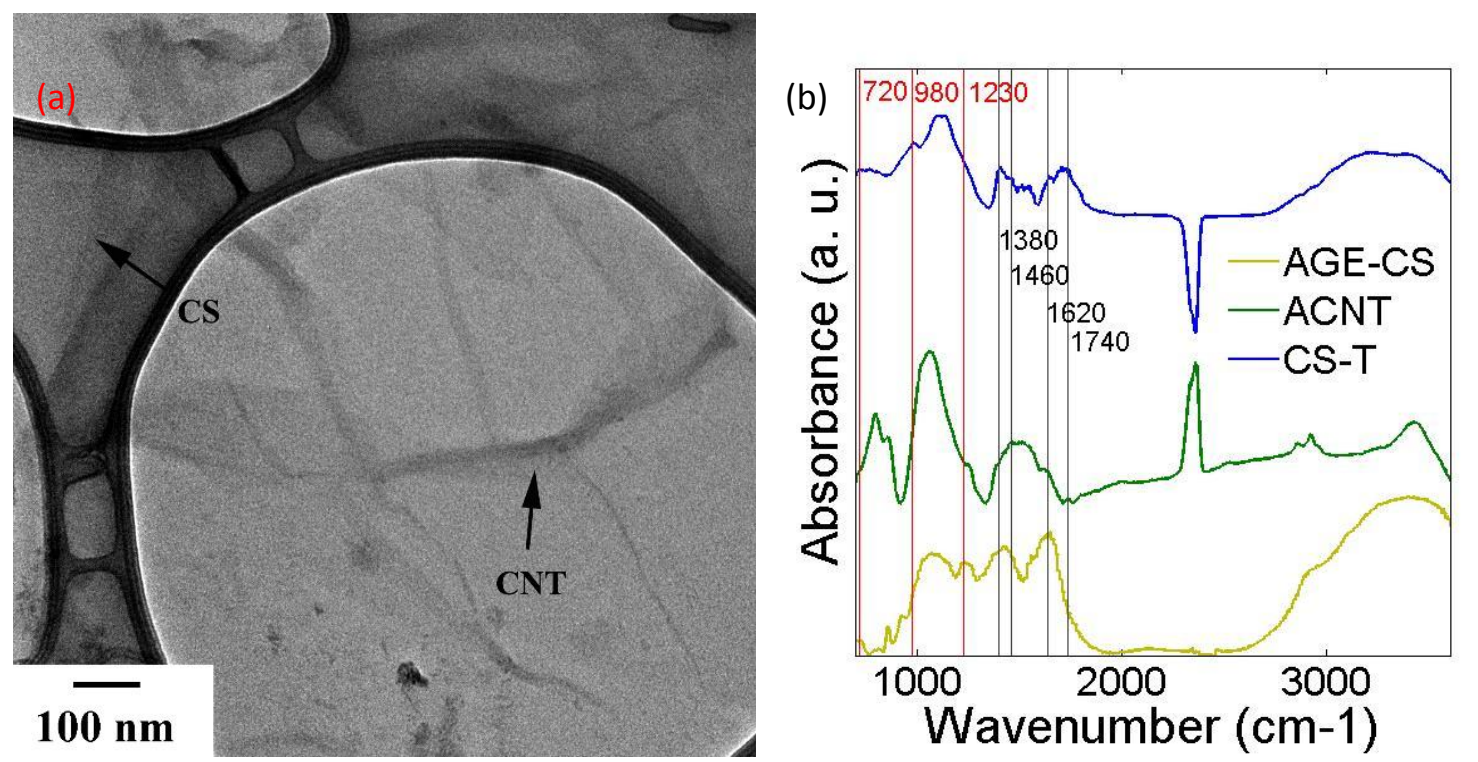

Fig. 4. The TEM images (a) and the FTIR spectrum (b) of CS-T.

\subsection{Mechanical Properties of DN gel}

The mechanical properties of the DN gel with different molecular stents were measured by the compression test as seen in Fig. 5. Small amount of the CS in the DN gel presents the lowest elongation-at-break and strength performance. This might be the result of CS leaking during the swelling in the AA solution which causes inhomogeneous AA monomer concentration in the hydrogel. The inhomogeneity of the N2 is causing from the leakage of the CS. This comes from the different swelling ratio at places with/without CS swelling when the N1 in the monomer solution of N2. The Control exhibited the smallest swelling ratio because of the very little charging of the N1 PAA. Due to the higher charging of the CS compared with the N1, the higher $\mathrm{N} 2$ monomer is absorbed at the place with the CS. Due to the inhomogeneous leaking of the CS, the final $\mathrm{N} 2$ network is not inhomogeneous. As the second network of PAA integrates the hydrogel, the inhomogeneous N2 PAA makes the DN gel vulnerable. 
The Yong's modulus of hydrogels is mainly come from the crosslink density. Due to the low crosslink density of the N2, samples has small swelling perform higher modulus, like the Control. On the other hand, the osmotic pressure comes from the CS can also provide mechanical support[35]. The higher concentration of the CS, the better mechanical properties hydrogel can achieve. The Yong's modulus of DN gel is from the balance of these two factors.

$\mathrm{D}(\mathrm{PCS})$ and $\mathrm{D}(\mathrm{CS}-\mathrm{T})$ show higher elongation-at-break as well as higher compressive strength than the D(CS). The uniformly dispersed PCS and CS-T became the molecular stent in the hydrogel, which entangled the N1 chains and improve the osmotic pressure. During the swelling in the monomer solution, the N1 was extended and became tough with the help of stents. Large amounts of the AA monomers flowed into the swollen N1 gel and form ductile N2 network during polymerization. This phenomenon results in a homogeneous structure which has tough first network and ductile second network. Although both the D(PCS) and D(CS-T) show high elongation-at-break and strength, the $\mathrm{D}(\mathrm{CS}-\mathrm{T})$ presented better results as its higher swelling degree ensured more AA monomer flowing to form homogeneous in the gel. The more homogeneous integration of the $\mathrm{D}(\mathrm{CS}-\mathrm{T})$ promises the better mechanical performance during test.
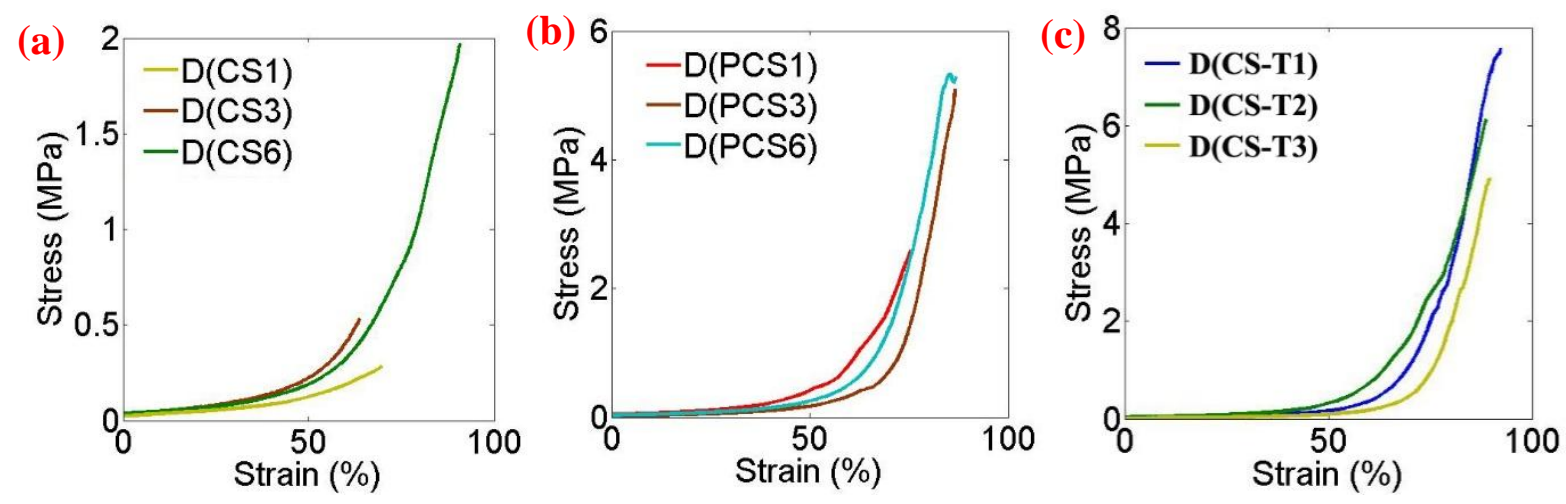

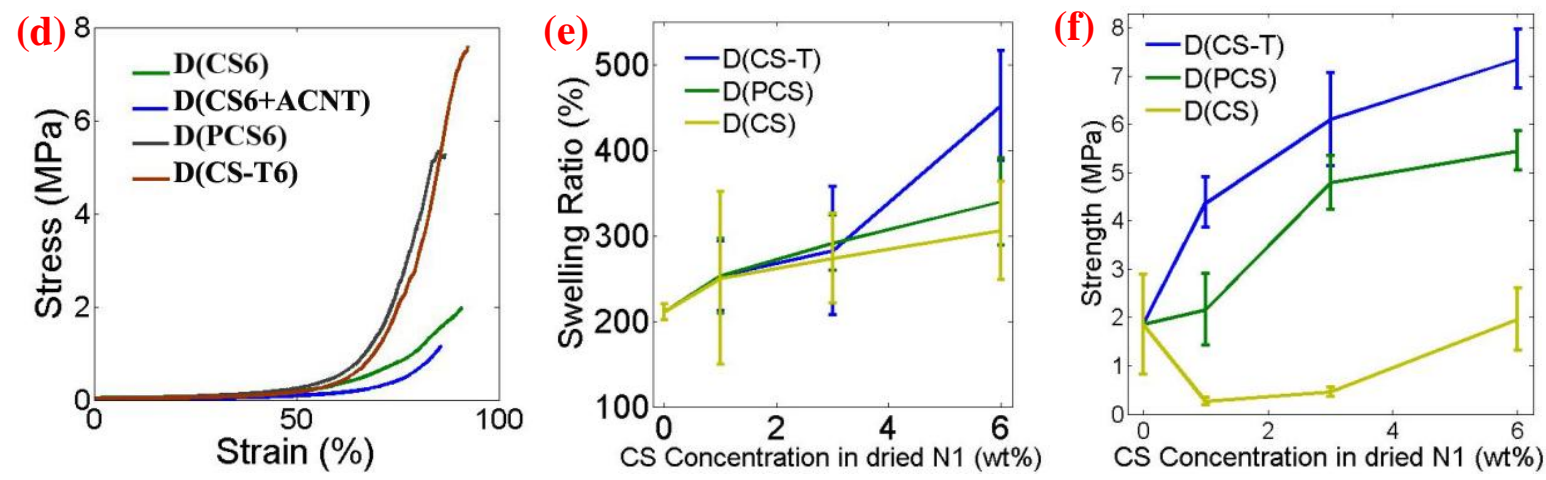

Fig. 5. The compression test of (a) D(CS), (b) D(PCS), and (c) D(CS-T). (d) The comparison of gels with different stents. The comparison of swelling degree (e) and strength (f) with different

DN gels.

Table 2. The swelling ratio, elongation-at-break, and strength of different DN gels.

\begin{tabular}{|c|c|c|c|c|c|}
\hline Sample & $\mathrm{Q}(\%)$ & $\begin{array}{c}\text { Elongation-at- } \\
\text { break (\%) }\end{array}$ & $\begin{array}{c}\text { Young's } \\
\text { Modulus (kPa) }\end{array}$ & $\begin{array}{c}\text { Strength } \\
(\mathrm{MPa})\end{array}$ & $\begin{array}{c}\text { Toughness } \\
\left(\mathrm{kJ} / \mathrm{m}^{3}\right)\end{array}$ \\
\hline CS-T6 & $452 \pm 64$ & $88 \pm 2.8$ & $131.6 \pm 7.0$ & $7.36 \pm 0.60$ & $1007 \pm 189$ \\
\hline CS-T3 & $282 \pm 75$ & $89 \pm 0.7$ & $98.5 \pm 16.3$ & $6.10 \pm 0.96$ & $887 \pm 156$ \\
\hline CS-T1 & $235 \pm 41$ & $89 \pm 0.5$ & $120.6 \pm 4.7$ & $4.38 \pm 0.52$ & $488 \pm 19$ \\
\hline PCS6 & $340 \pm 51$ & $86 \pm 1.1$ & $97.3 \pm 19.9$ & $5.45 \pm 0.40$ & $740 \pm 68$ \\
\hline PCS3 & $291 \pm 32$ & $86 \pm 1.4$ & $100.4 \pm 6.3$ & $4.79 \pm 0.55$ & $518 \pm 54$ \\
\hline PCS1 & $253 \pm 44$ & $79 \pm 0.5$ & $85.3 \pm 13.9$ & $2.16 \pm 0.75$ & $368 \pm 41$ \\
\hline CS6 & $306 \pm 57$ & $91 \pm 1.2$ & $109.1 \pm 6.4$ & $1.97 \pm 0.64$ & $361 \pm 15$ \\
\hline CS3 & $274 \pm 52$ & $72 \pm 2.2$ & $119.1 \pm 15.2$ & $0.46 \pm 0.09$ & $94 \pm 13$ \\
\hline CS1 & $250 \pm 101$ & $65 \pm 4.3$ & $122 \pm 31.7$ & $0.26 \pm 0.08$ & $74 \pm 28$ \\
\hline CS6+ACNT & $388 \pm 88$ & $83 \pm 10.1$ & $74.1 \pm 28.4$ & $1.75 \pm 0.69$ & $146 \pm 21$ \\
\hline Control & $211 \pm 9$ & $84 \pm 0.6$ & $150.6 \pm 7.2$ & $1.86 \pm 1.03$ & $219 \pm 49$ \\
\hline
\end{tabular}

\subsection{Leaking}

The leaking of the CS6, PCS6 and CS-T6 in N1 gel was analyzed by UV absorption spectra. The peak intensity of the CS around $260 \mathrm{~nm}$ was used to measure the relative concentration of the CS in $50 \mathrm{ml}$ swelling solution. As can be seen in the Fig. 6, 15 times more of the CS were leaked from the S(CS6) and S(PCS6) compared with the S(CS-T6) after 7 days of 
swelling. These results indicated that the large structure of the CS-T can efficiently entangle with the N1 and prevent the leaking.
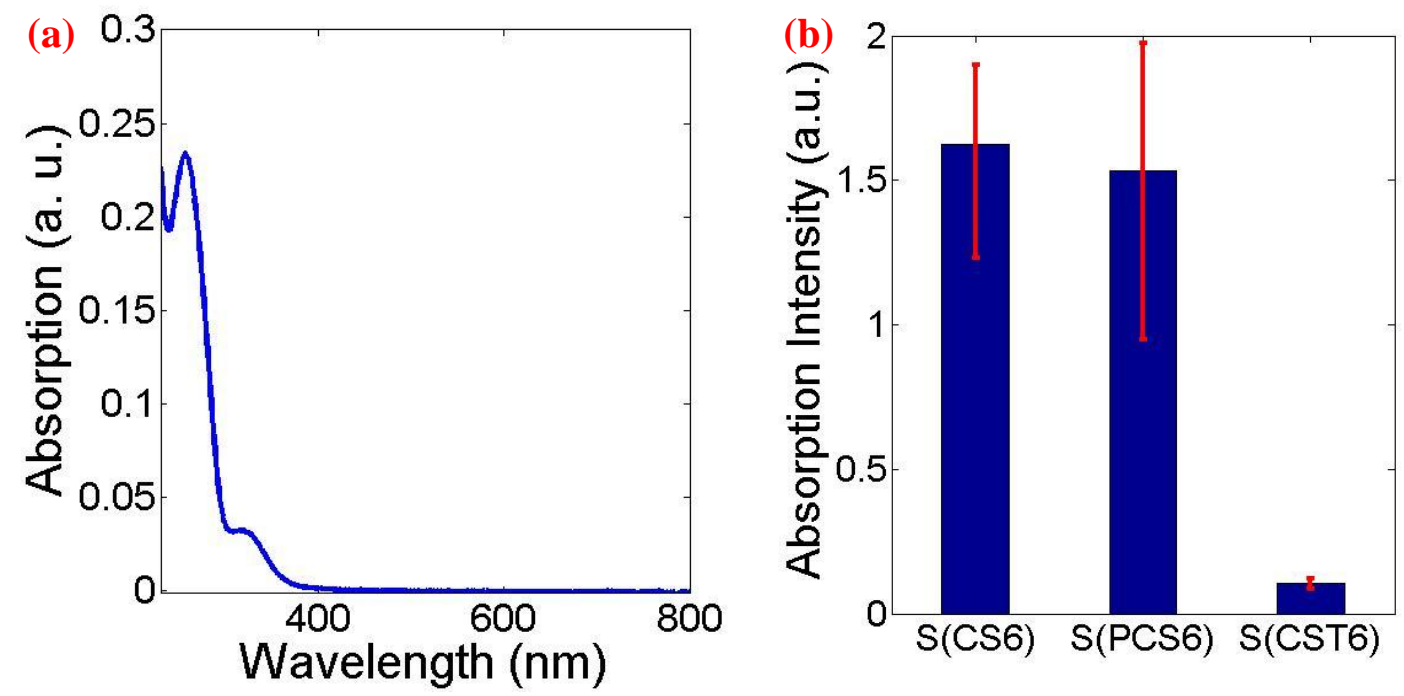

Fig. 6. (a) The UV-vis spectrum of CS in water, and (b) The amount of CS leaked from S(CS6), S(PCS6), and S(CS-T6) after 7 days of swelling.

\subsection{Biocompatibility}

Cell culture experiments with U87-MG cells were carried out to evaluate the biocompatibility of the hydrogels. Proliferations at 24,48 , and $72 \mathrm{~h}$ after culturing cells with the Control and D(CS-T6) are shown in Fig. 7. The D(CS-T6) supported proliferations of U87-MG cells, while fewer cells were detected after culturing with Control. Also, the proliferative rate of $\mathrm{D}$ (CS-T6) is 10 times higher than the Control (17120 cell/hr of VBDN to 1760 cell/hr of BDN). These results indicated that the $\mathrm{D}(\mathrm{CS}-\mathrm{T} 6)$ has a better biocompatibility than the Control due to the existence of CS-T. 


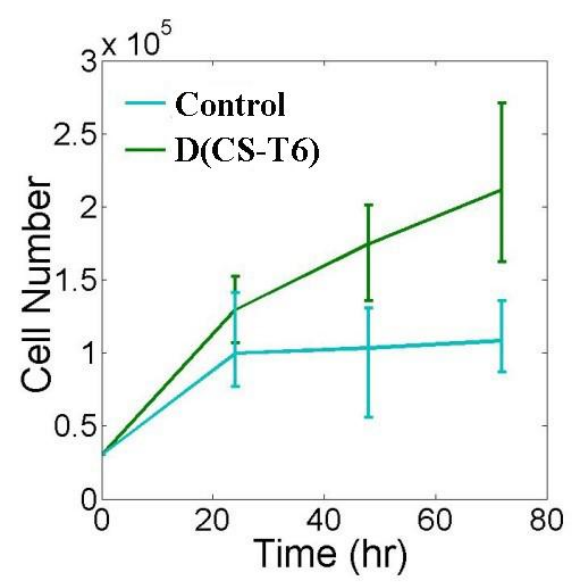

Fig. 7. The biocompatibility tests of Control and D(CS-T6).

\section{Conclusions}

In this work, a biomimetic structure (CS-T) was synthesized to perform as a molecular stent in double network hydrogel. Similar with the proteoglycans, the CS-T has a backbone carbon nanotube anchors large amount of chondroitin sulfate chains. With the aid of CS-T, double network hydrogel made by neutral polymers performs high mechanical properties. This achievement not only presented an affordable way to synthesis proteoglycans, but also ensures tough hydrogel with different functions by using the most suitable polymer.

Acknowledgement

The authors would like to thank Imaging Center in Texas Tech University to allow us using their equipment. The authors would like to acknowledge the supporting from NSF grant (number 1228127).

Reference:

[1] K.Y. Lee, D.J. Mooney, Hydrogels for tissue engineering, Chem. Rev., 101 (2001) 1869-1879.

[2] L. Dong, A.K. Agarwal, D.J. Beebe, H.R. Jiang, Adaptive liquid microlenses activated by stimuliresponsive hydrogels, Nature, 442 (2006) 551-554. 
[3] J.P. Gong, Friction and lubrication of hydrogels - its richness and complexity, Soft Matter, 2 (2006) 544-552.

[4] N.G. Kandile, A.S. Nasr, Environment friendly modified chitosan hydrogels as a matrix for adsorption of metal ions, synthesis and characterization, Carbohyd. Polym., 78 (2009) 753-759.

[5] J. Wang, J. Qiu, S. Wang, 3D Core-Shell Simulation of Hydrogel Swelling Behavior for Controlled Drug Delivery, in: ASME 2013 International Mechanical Engineering Congress and Exposition, American Society of Mechanical Engineers, San Diego, California, USA, 2013, pp. V03BT03A027-V003BT003A027. [6] J.A. Burdick, G.D. Prestwich, Hyaluronic Acid Hydrogels for Biomedical Applications, Adv. Mater., 23 (2011) H41-H56.

[7] J.A. Rowley, G. Madlambayan, D.J. Mooney, Alginate hydrogels as synthetic extracellular matrix materials, Biomaterials., 20 (1999) 45-53.

[8] Y.H. Gong, L.J. He, J. Li, Q.L. Zhou, Z.W. Ma, C.Y. Gao, J.C. Shen, Hydrogel-filled polylactide porous scaffolds for cartilage tissue engineering, Journal of Biomedical Materials Research Part B-Applied Biomaterials, 82B (2007) 192-204.

[9] Y.D. Park, N. Tirelli, J.A. Hubbell, Photopolymerized hyaluronic acid-based hydrogels and interpenetrating networks, Biomaterials., 24 (2003) 893-900.

[10] J.Y. Sun, X.H. Zhao, W.R.K. Illeperuma, O. Chaudhuri, K.H. Oh, D.J. Mooney, J.J. Vlassak, Z.G. Suo, Highly stretchable and tough hydrogels, Nature, 489 (2012) 133-136.

[11] C.W. Peak, J.J. Wilker, G. Schmidt, A review on tough and sticky hydrogels, Colloid Polym. Sci., 291 (2013) 2031-2047.

[12] T. Nakajima, T. Kurokawa, H. Furukawa, Q.M. Yu, Y. Tanaka, Y. Osada, J.P. Gong, Super Tough Gels with a Double Network Structure, Chinese. J. Polym. Sci., 27 (2009) 1-9.

[13] J.P. Gong, Y. Katsuyama, T. Kurokawa, Y. Osada, Double-network hydrogels with extremely high mechanical strength, Adv. Mater., 15 (2003) 1155-+.

[14] Y.H. Na, T. Kurokawa, Y. Katsuyama, H. Tsukeshiba, J.P. Gong, Y. Osada, S. Okabe, T. Karino, M. Shibayama, Structural characteristics of double network gels with extremely high mechanical strength, Macromolecules, 37 (2004) 5370-5374.

[15] T. Nakajima, H. Furukawa, Y. Tanaka, T. Kurokawa, Y. Osada, J.P. Gong, True Chemical Structure of Double Network Hydrogels, Macromolecules, 42 (2009) 2184-2189.

[16] J.L. Wang, J.H. Wei, S.H. Su, J.J. Qiu, S.R. Wang, lon-linked double-network hydrogel with high toughness and stiffness, J. Mater. Sci., 50 (2015) 5458-5465.

[17] J. Wei, J. Wang, S. Su, S. Wang, J. Qiu, Tough and fully recoverable hydrogels, J. Mater. Chem. B, 3 (2015) 5284-5290.

[18] J.P. Gong, Why are double network hydrogels so tough?, Soft Matter, 6 (2010) 2583-2590.

[19] W. Yang, H. Furukawa, J.P. Gong, Highly Extensible Double-Network Gels with Self-Assembling Anisotropic Structure, Adv. Mater., 20 (2008) 4499-4503.

[20] X. Lu, C.Y. Chan, K.I. Lee, P.F. Ng, B. Fei, J.H. Xin, J. Fu, Super-tough and thermo-healable hydrogel promising for shape-memory absorbent fiber, J. Mater. Chem. B, 2 (2014) 7631-7638.

[21] G. Du, G. Gao, R. Hou, Y. Cheng, T. Chen, J. Fu, B. Fei, Tough and Fatigue Resistant Biomimetic Hydrogels of Interlaced Self-Assembled Conjugated Polymer Belts with a Polyelectrolyte Network, Chem. Mater., 26 (2014) 3522-3529.

[22] T.C. Suekama, J. Hu, T. Kurokawa, J.P. Gong, S.H. Gehrke, Double-Network Strategy Improves Fracture Properties of Chondroitin Sulfate Networks, Acs Macro Letters, 2 (2013) 137-140.

[23] T. Nakajima, H. Sato, Y. Zhao, S. Kawahara, T. Kurokawa, K. Sugahara, J.P. Gong, A Universal Molecular Stent Method to Toughen any Hydrogels Based on Double Network Concept, Adv. Funct. Mater., 22 (2012) 4426-4432. 
[24] Y. Zhao, T. Nakajima, J.J. Yang, T. Kurokawa, J. Liu, J. Lu, S. Mizumoto, K. Sugahara, N. Kitamura, K. Yasuda, A.U.D. Daniels, J.P. Gong, Proteoglycans and Glycosaminoglycans Improve Toughness of Biocompatible Double Network Hydrogels, Adv. Mater., 26 (2014) 436-442.

[25] S. Sarkar, S.E. Lightfoot-Vidal, C.L. Schauer, E. Vresilovic, M. Marcolongo, Terminal-end functionalization of chondroitin sulfate for the synthesis of biomimetic proteoglycans, Carbohydr Polym, 90 (2012) 431-440.

[26] G.T. Hermanson, Bioconjugate Techniques, Academic Press, 2013.

[27] J.H. Wei, J.J. Qiu, Allyl-Functionalization enhanced thermally stable graphene/fluoroelastomer nanocomposites, Polymer, 55 (2014) 3818-3824.

[28] G.L. Du, G.R. Gao, R.X. Hou, Y.J. Cheng, T. Chen, J. Fu, B. Fei, Tough and Fatigue Resistant Biomimetic Hydrogels of Interlaced Self-Assembled Conjugated Polymer Belts with a Polyelectrolyte Network, Chem. Mater., 26 (2014) 3522-3529.

[29] N. Chiaoprakobkij, N. Sanchavanakit, K. Subbalekha, P. Pavasant, M. Phisalaphong, Characterization and biocompatibility of bacterial cellulose/alginate composite sponges with human keratinocytes and gingival fibroblasts, Carbohyd. Polym., 85 (2011) 548-553.

[30] M. Foot, M. Mulholland, Classification of chondroitin sulfate A, chondroitin sulfate $C$, glucosamine hydrochloride and glucosamine 6 sulfate using chemometric techniques, J Pharm Biomed Anal, 38 (2005) 397-407.

[31] F. Cabassi, B. Casu, A.S. Perlin, Infrared absorption and raman scattering of sulfate groups of heparin and related glycosaminoglycans in aqueous solution, Carbohyd. Res., 63 (1978) 1-11.

[32] J.M. Huang, H.J. Huang, Y.X. Wang, W.Y. Chen, F.C. Chang, Preparation and Characterization of Epoxy/Polyhedral Oligomeric Silsesquioxane Hybrid Nanocomposites, J. Polym. Sci. Pol. Phys, 47 (2009) 1927-1934.

[33] L. Stobinski, B. Lesiak, L. Kover, J. Toth, S. Biniak, G. Trykowski, J. Judek, Multiwall carbon nanotubes purification and oxidation by nitric acid studied by the FTIR and electron spectroscopy methods, J. Alloy. Compd., 501 (2010) 77-84.

[34] U.J. Kim, C.A. Furtado, X.M. Liu, G.G. Chen, P.C. Eklund, Raman and IR spectroscopy of chemically processed single-walled carbon nanotubes, J. Am. Chem. Soc., 127 (2005) 15437-15445.

[35] G. Vunjak - Novakovic, I. Martin, B. Obradovic, S. Treppo, A. Grodzinsky, R. Langer, L. Freed, Bioreactor cultivation conditions modulate the composition and mechanical properties of tissue engineered cartilage, J. Orthopaed. Res., 17 (1999) 130-138. 